\title{
МРНТИ 28.15.15
}

УДК 681.5.037.26

https://doi.org/10.51889/2020-3.1728-7901.07

\author{
К.С. Дальбекова ${ }^{l}$, Ф.Р. Гусманова ${ }^{2}$ С.Б. Беркимбаева ${ }^{1}$, А.К. Искакова ${ }^{l}$ \\ ${ }^{l}$ Университет международного бизнеса, г. Алматы, Казахстан \\ ${ }^{2}$ Казахский национальный университет имени аль-Фараби, г. Алматы, Казахстан
}

\section{ПРОБЛЕМЫ УСТОЙЧИВОСТИ ЛИНЕЙНЫХ НЕСТАЦИОНАРНЫХ СИСТЕМ НА КОНЕЧНОМ ОТРЕЗКЕ ВРЕМЕНИ}

\begin{abstract}
Аннотация
При изучении различных процессов происходящих в реальной действительности, приходится сталкиваться с одним из наиболее важных понятий - понятием об устойчивости движения. Основы теории устойчивости движения были разработаны в конце прошлого века великим русским ученым А.М. Ляпуновым. Как известно, устойчивость по Ляпунову рассматривается на бесконечном интервале времени, что является серьезным препятствием для многих приложений, т.к. большинство объектов исследования функционируют в течение конечного промежутка времени. Понятие устойчивости, введенное для неограниченного промежутка времени, не может быть использовано для оценки свойств движения в пределах конечного промежутка времени. Исследование устойчивости движения путем анализа решений соответствующих уравнений допустимо и имеет смысл лишь при условии полной адекватности математической модели физической реальности. Цель работы заключается в исследовании устойчивости и стабилизации движения линейных нестационарных систем на конечном интервале времени.
\end{abstract}

Ключевые слова: устойчивость движения, линейные нестационарные системы, конечный отрезок времени, фундаментальная матрица, дифференциальные уравнения возмущенного движения, след матрицы.

\author{
Аңудатпа \\ К.С. Дальбекова ${ }^{1}$ Ф.Р. Гусманова ${ }^{2}$ С.Б. Беркімбаева ${ }^{1}$, А.К Искакова \\ ${ }^{1}$ Хальққаральқ бизнес университеті, Алматьл қ., Қазақстан \\ ${ }^{2}$ Әл-Фараби атындавы Қазақ ұлттық университеті, Алматы қ., Қазақстан \\ ШЕКТЕУЛІ УАҚЫТ АРАЛЫҒЫНДАҒЫ СТАЦИОНАРЛЫ ЕМЕС \\ ЖҮЙЕЛЕРДІН ТҰРАҚТЫЛЫҚ МӘСЕЛЕЛЕРІ
}

Нақты болып жатқан әртүрлі үрдістерді зерттеу кезінде маңызды ұғымдардың бірі-қозғалыс тұрақтылығы туралы ұғымымен кездесуіміз мүмкін. Қозғалыс тұрақтылығы теориясының негіздерін өткен ғасырдың соңында ұлы орыс ғалымы А.М. Ляпунов жасаған. Өзімізге белгілі, Ляпуновтың тұрақтылығы шексіз уақыт аралығында қарастырылады, бұл көптеген қосымшалар үшін үлкен кедергі болып табылады, өйткені зерттеу объектілерінің көпшілігі шектеулі уақыт аралығында жұмыс істейді. Шексіз уақыт аралығында енгізілген тұрақтылық ұғымын қозғалыс қасиеттерін шектеулі уақыт аралығында бағалау үшін қолдануға болмайды. Тиісті теңдеулердің шешімдерін талдау арқылы қозғалыс тұрақтылығын зерттеу физикалық нақтылықтың математикалық моделінің толық жеткіліктілігі жағдайында ғана қолайлы және мағыналы болады. Жұмыстың мақсаты-сызықты емес жүйелердің қозғалысының тұрақтытылығын шектеулі уақыт аралығында зерттеу.

Түйін сөздер: қозғалыс тұрақтылығы, сызықты стационарлық емес жүйелер, шектеулі ауқыт аралығы, іргелі матрица, ауытқу қозғалысының дифференциалдық теңдеулері, матрицаның ізі.

\section{Abstract \\ PROBLEMS OF STABILITY OF LINEAR NON-STATIONARY SYSTEMS ON A FINITE TIME INTERVAL \\ ${ }^{1}$ Dalbekova K.S. ${ }^{1}$, Gusmanova F.R. ${ }^{2}$, Berkimbaeva S.B. ${ }^{l}$, Iskakova A.K. ${ }^{1}$ \\ ${ }^{1}$ University of international business, Almaty, Kazakhstan \\ ${ }^{2}$ Kazakh National University named after al-Farabi, Almaty, Kazakhstan}

When studying various processes taking place in real life, we have to deal with one of the most important concepts the concept of stability of movement. The foundations of the theory of stability of motion were developed at the end of the last century by the great Russian scientist A. M. Lyapunov. As is known, Lyapunov stability is considered on an infinite time interval, which is a serious obstacle for many applications, since most of the objects of research function for a finite period of time. The concept of stability, introduced for an unlimited period of time, cannot be used to evaluate the properties of motion within a finite period of time. The study of motion stability by analyzing solutions of the corresponding equations is permissible and makes sense only if the mathematical model of physical reality is fully 
adequate. The purpose of this work is to study the stability and stabilization of the motion of linear non-stationary systems.

Keywords: stability of motion, linear non-stationary systems, finite time interval, fundamental matrix, differential equations of perturbed motion, matrix trace.

Задача об устойчивости реальных процессов обычно сводится к исследованию решений некоторых систем дифференциальных, интегро-дифференциальных или другого типа уравнений. Исследование устойчивости движения путем анализа решений соответствующих уравнений допустимо и имеет смысл лишь при условии полной адекватности математической модели физической реальности. К исследованию нестационарных линейных систем обыкновенных дифференциальных уравнений приводят многие задачи механики и техники, в частности, задачи анализа систем и навигации движущихся объектов. Хотя теория нестационарных линейных систем в силу практической необходимости интенсивно разрабатывается, многообразие возможных зависимостей коэффициентов системы от времени не позволило пока создать достаточно конструктивную теорию для нестационарных линейных систем общего вида [1-3].

Но, даже если, адекватность математической модели физической реальности соблюдается при всех $t>t_{0}$, это еще не означает, что между понятием устойчивости на конечном и неограниченном промежутках времени возможно установить взаимооднозначное соответствие.

Рассмотрим два дифференциальных уравнения [4]:

$$
\frac{d x}{d t}=f_{1}(t, x), \quad \frac{d x}{d t}=f_{2}(t, x),
$$

правые части которых соответствуют условиям

$$
\begin{gathered}
f_{i}(t, x)=0, \quad i=1,2 \\
f_{1}(t, x)=f_{2}(t, x) \text { при } t \in\left[t_{0}, T\right] .
\end{gathered}
$$

В силу свойств правых частей решения этих двух уравнений в пределах конечного промежутка времени $t_{0} \leq t<T$ совпадают. Поэтому, если эти два уравнения представляют собой два разных процесса, то на конечном промежутке времени $\left[t_{0} T\right)$ эти процессы могут быть либо одновременно устойчивы, либо одновременно неустойчивы [5]. Но может случиться, что, например, тривиальное решение первого уравнения устойчиво по Ляпунову, а тривиальное решение второго уравнения неустойчиво. Поскольку решение задачи устойчивости по Ляпунову определяется свойствами функций $f_{1}$ и $f_{2}$ на промежутке, а при $t>T$ эти функции, тождественно совпадающие на конечном отрезке времени $\left[t_{0} T\right)$, могут отличаться друг от друга как угодно. Соображения такого рода определяют необходимость введения понятия устойчивости процесса на конечном отрезке времени.

Наличие зависимости коэффициентов системы от времени вносит принципиальные трудности в изучении структурных свойств системы (устойчивости, управляемости, и наблюдаемости). Исследование устойчивости линейных нестационарных систем на конечном отрезке времени, обеспечивающее точное попадание к началу координат за конечное время, а также на бесконечном интервале времени до сих пор полностью нерешенная задача.

Исследуем устойчивость движения линейных нестационарных систем на конечном интервале времени.

Рассмотрим линейную систему дифференциальных уравнений:

$$
\frac{d x}{d t}=A(t) x, \quad x\left(t_{0}\right)=x_{0}, \quad t \in\left[t_{0}, T\right)
$$

где $x-n$-мерный вектор состояния системы,

$$
A(t) \in\left[t_{0}, T\right) \text { - матрица размера } n \times n, \text { причем }
$$




$$
\lim _{t \rightarrow T}\|A(t)\|=\infty
$$

Пусть $\Phi(t)$ - фундаментальная матрица решений системы (1). Тогда матрицу $\Phi(t)$ можно определить из матричного дифференциального уравнения дифференциального уравнения [6].

$$
\frac{d x}{d t} \Phi(t)=A(t) \Phi x, \quad \Phi\left(t_{0}\right)=E_{n}
$$

Тогда матрица

$$
\Phi(\mathrm{t}, \tau)=\Phi(\mathrm{t}) \Phi^{-1}(\tau)
$$

может быть определена как решение матричного дифференциального уравнения вида:

$$
\frac{d x}{d t} \Phi(t, \tau)=A(t) \Phi(t, \tau), \quad \Phi(t, \tau)=E_{n}
$$

Теорема 1. Для уравнения возмущенного движения

$$
\frac{d x}{d t}=X(t, x), \quad t \in\left[t_{0}, T\right),
$$

где $x=\left(x_{1}, x_{2}, \ldots, x_{n}\right)^{*}, X(t, x)=\left(X_{1}(t, x), X_{2}(t, x), \ldots, X_{n}(t, x)\right)^{*}$

с нормой $\|x\|=\left(\sum_{j=1}^{n} x_{j}^{2}\right)^{\frac{1}{2}} \quad$ существует T - определенно-положительная функция $V(t, x) \in C_{t x}^{(1,1)}\left(Z_{0}\right)$, допускающая бесконечно большой низший предел (ББНП) при $t \rightarrow T$ и с знакоотрицательной полной производной по времени $t$, то положение равновесия $x=0$ устойчиво на конечном отрезке времени [3], [7].

Следствие 1. Если дифференциальные уравнения возмущенного движения (4) таковы, что существует Т-определенно - положительная функция $V(t, x)$, где $V(t, a)$ монотонно-возрастающая по $t$ от $[0, \infty)$, а полная производная по времени $\dot{V}$ является знакоотрицательной Z, то положение равновесия $x=0$ устойчиво на конечном отрезке времени (КОВ) [4], [8].

Следствие 2. Пусть для системы (4) $T=\infty$. Если дифференциальные уравнения возмущенного движения таковы, что существует определенно - положительная функция $V(t, x)$ такая, что для любого ненулевого вектора $a \in R_{\text {функция }} V(t, a)$ монотонно - возрастющая по $t$

$$
\lim _{t \rightarrow T} V(t, a)=\infty,
$$

а полная производная по времени $t$ от функции $V$ является знакоотрицательной, то положение равновесия $x=0$ асимптотически устойчиво по Ляпунова.

Замечание. Как известно, в работе [9] предложено перевести конечный интервал по $t$ в интервал $[0, \infty)$ по $\tau$ с помощью преобразования

$$
\tau=\frac{1}{1-t}-1
$$

Исходные уравнения на интервале [0,1] будут иметь вид:

$$
\frac{d x}{d \tau}=\frac{1}{(1+\tau)^{2}} X(\tau, x), \quad \tau \in[0, \infty)
$$




$$
\frac{d V}{d \tau}=\frac{1}{(\tau+1)^{2}} \frac{d V}{d t}
$$

но устойчивость при этом не является асимптотической.

Поэтому условие $\lim _{t \rightarrow \infty}\|x(t)\|=0$.

Для исследования устойчивости на конечном отрезке времени рассмотрим фукцию Ляпунова вида [10]:

$$
V(t, x(t))=x^{*} K(t) x, \quad t \in\left[t_{0}, T\right)
$$

Полная производная по $t$ от функции в силу системы (1)

$$
\frac{d v}{d t}=x^{*}\left[\dot{K}(t)+K(t) A(t)+A^{*}(t) K(t)\right] x, \quad t \in\left[t_{0}, T\right)
$$

и $\dot{V}=0$ тогда и только тогда, когда

$$
\dot{K}(t)+K(t) A(t)+A^{*}(t) K(t)=0, \quad t \in\left[t_{0}, T\right), \quad K\left(t_{0}\right)=E_{n}
$$

Решением этой системы является

$$
K^{-1}(t)=\Phi(t) \Phi^{*}(t), \quad t \in\left[t_{0}, T\right)
$$

Где $\Phi(t)=\Phi\left(t, t_{0}\right)$ фундаментальная матрица решений системы (1).

Следовательно

$$
V(t, x(t))=x^{*}\left[\Phi(t) \Phi^{*}(t)\right]^{-1} x, \quad t \in\left[t_{0}, T\right)
$$

T- определенно-положительная и допускает бесконечно большой низший предел при $t \rightarrow T[10]$, если

$$
\lim _{t \rightarrow T} \Phi_{i j}(t)=0, \quad \forall i, j=1, n
$$

Так как

$$
\Phi^{-1}(t)=\frac{\operatorname{adj} A(t)}{\operatorname{det} A(t)}
$$

Тогда с учетом равенств

$$
\begin{gathered}
\operatorname{det} \Phi(t)=\operatorname{det} A(t)=e^{\int_{t_{0}}^{t} S p A(\tau) d \tau} \\
S p A(t)=\sum_{j=1}^{n} a_{j j}(t)
\end{gathered}
$$

Получим следующее утверждение:

Теорема 2. Положение равновесия $x=0$ линейной нестационарной системы (1) устойчиво на конечном отрезке времени, если матрица $A(t)$ гурвицева для $\forall t \in\left[t_{0}, T\right)$ и выполняется

$$
\lim _{t \rightarrow T} \int_{t_{0}}^{t} \operatorname{SpA}(\tau) d \tau=\infty
$$


Доказательство: Заметим, что условие (11) равносильно или достаточно для выполнения соотношения (9). Рассмотрим уравнение (7), для которой справедливо $K^{-1}(T)=0$.

Функция $V(t, a)=a^{*} K(t) a$

должна быть монотонно возрастающей. Такое возможно в том случае, если

$\dot{K}(t)>0$, так как, при этом

$$
x^{*} K\left(t_{1}\right) x<x^{*} K\left(t_{2}\right) x, \quad t_{1}<t_{2} .
$$

Согласно уравнению (6) при $K(t)>0$, то есть при положительно определенном $K(t)$ достаточно выполнения неравенства [11]:

$$
K(t) A(t)+A^{*}(t) K(t)<0 \quad t \in\left[t_{0}, T\right)
$$

имеет единственное решение, если матрица $A(t)$ гурвицева $\forall t \in\left[t_{0}, T\right)$.

В работе получены достаточные условия устойчивости линейных нестационарных систем на конечном отрезке времени.

Исследуем устойчивость на конечном отрезке времени с помощью теоремы 2, то есть проверим выполнение условия (11) на примере следующего скалярное уравнение

$$
\frac{d x}{d t}=\frac{e^{-2}+e^{-2 t}}{e^{-2}-e^{-2 t}} x, \quad x(0)=x_{0}, \quad t \in[0,1]
$$

где $(t, x)=(1, x(1)=0)$ является особой точкой типа $\left(\frac{0}{0}\right)$

Тогда имеем

$$
\begin{gathered}
\lim _{t \rightarrow 1} \int_{0}^{t} \frac{e^{-2}+e^{-2 \tau}}{e^{-2}-e^{-2 \tau}} d t=\lim _{t \rightarrow 1} \int_{0}^{t}\left(1+\frac{2 e^{-2 \tau}}{e^{-2}-e^{-2 \tau}}\right) d t= \\
=\lim _{t \rightarrow 1} \int_{0}^{t}\left[\left(t+\ln \left|e^{-2}-e^{-2 \tau}\right|\right)_{0}^{t} \mid\right]=1+\ln 0-\ln \left(e^{-2}-1\right)=-\infty
\end{gathered}
$$

Следовательно, тривиальное решение уравнения (13) устойчиво на конечном отрезке времени. Действительно, решение уравнения

$$
x(t)=\frac{e^{t}\left(e^{-2 t}-e^{-2}\right) x_{0}}{1-e^{-2}}, \quad t \in[0,1]
$$

Обладает свойством $x(1)=0$

Рассмотрим выполнение условия теоремы 1 на следующем примере:

$$
\begin{aligned}
& \frac{d x_{1}}{d t}=-\frac{x_{1}}{1-t}-\left(1-t^{2}\right) x_{1}^{2} x_{2}^{2} \\
& \frac{d x_{2}}{d t}=-\frac{x_{2}}{1-t}, \quad t \in[0,1)
\end{aligned}
$$

с начальным условием $x_{1}(0)=1, \quad x_{2}(0)=1$.

Возьмем функцию Ляпунова вида

$$
V(t, x)=\frac{K(t)}{2}\left(x_{1}^{2} x_{2}^{2}\right), \quad t \in[0,1)
$$

и вычислим 


$$
\dot{V}=\left(\frac{K(t)}{2}-\frac{K(t)}{1-t}\right)\left(x_{1}^{2}+x_{2}^{2}\right)-K(t)(1-t)^{2} x_{1}^{2} x_{2}^{2}, \quad t \in[0,1)
$$

тогда $\quad \dot{V}=-K(t)(1-t)^{2} x_{1}^{2} x_{2}^{2}$,

Если $\left(\frac{K(t)}{2}-\frac{K(t)}{1-t}\right)=0, \quad t \in[0,1)$

При этом в частности

$$
K(t)=\frac{1}{(1-t)^{2}}>0
$$

Следовательно, функция $V(t, x)$ имеет вид:

$$
\begin{array}{cc}
V(t, x)=\frac{x_{1}^{2} x_{2}^{2}}{(1-t)^{2}} & t \in[0,1) \\
\lim _{t \rightarrow 1} V(t, x)=\lim _{t \rightarrow 1} \frac{a_{1}^{2}+a_{2}^{2}}{(1-t)^{2}}=\infty, \quad \text { при }\|a\| \neq 0
\end{array}
$$

Таким образом, все условия теоремы 1 выполняются и положение равновесия $x=0$ устойчиво на конечном отрезке времени. Действительно, решение системы

$$
\begin{gathered}
x_{1}(t)=(1-t) \exp \left(\frac{(1-t)^{5}-1}{5}\right) \\
x_{2}(t)=(1-t), \quad t \in[0,1)
\end{gathered}
$$

обладает свойством $x_{1}(1)=0, x_{2}(1)=0$.

Список использованной литературь:

1 Ляпунов А.М. Общая задача об устойчивости движения. - М., Гостехиздат, 1950.

2 Меркин Д.Р. Введение в теорию устойчивости движения. С.-П., М., Краснодар: Лань, 2003.

3 Бияров Т.Н. Об устойчивости нелинейных систем на КОВ. - 1988.

4 Бияров Т.Н., Дальбекова К.С. Устойчивость на конечном отрезке времени линейных нестационарных систем. // Известия НАН РК, серия физ. -мат., 1993, №5 (174)

5 Беллман Р. Теория устойчивости решений дифференциальных уравнений. -М.: ИЛ, 1954

6 Абгарян К.А. Матричные и асимптотические методы в теории линейных систем.-М.: Наука, 1973.$431 \mathrm{c}$.

7 Абгарян К.А. К проблеме устойчивости линейных нестационарных систем // ДАН СССР.- 1989.-T. 308, вып. 6.

8 Айсагалиев С.А. Анализ и синтез автономных нелинейных систем автоматического управления. - АлмаАта, Наука КазССР, 1980.-244 с.

9 Летов А.М. Устойчивость нелинейных регулируемых систем. -М.: Физматгиз, 1962.-403 c.

10 Малкин И.Г. Теория устойчивости движения.-URSS.:-Изд.4,2017.-432c.

11 Федорюк М.В.Асимптотические методы для линейных обыкновенных дифференциальных уравнений.URSS.: Либроком, 2015.-390с.

\section{References}

1 Ljapunov A.M. (1950) Obshhaja zadacha ob ustojchivosti dvizhenija [the General problem of stability of motion]. Gostehizdat. (In Russian)

2 Merkin D.R. (2003) Vvedenie v teoriju ustojchivosti dvizhenija [introduction to the theory of stability of motion]. Krasnodar: Lan'. (In Russian)

3 Bijarov T.N. (1988) Ob ustojchivosti nelinejnyh sistem na KOV [On stability of nonlinear systems in COVE]. (In Russian) 
4 Bijarov T.N., Dal'bekova K.S.(1993) Ustojchivost' na konechnom otrezke vremeni linejnyh nestacionarnyh system [Stability on a finite time interval linear nonstationary systems]. Izvestija NAN RK, serija fiz.-mat, №5 (174). (In Russian)

5 Bellman R. (1954) Teorija ustojchivosti reshenij differencial'nyh uravnenij [Theory of stability of solutions of differential equations]. M.: IL. (In Russian)

6 Abgarjan K.A. (1973) Matrichnye i asimptoticheskie metody v teorii linejnyh system [Matrix and asymptotic methods in the theory of linear systems]. M.: Nauka. 431. (In Russian)

7 Abgarjan K.A. (1989) K probleme ustojchivosti linejnyh nestacionarnyh system [On the problem of stability of linear non-stationary systems]. DAN SSSR.-T. 308, 6. (In Russian)

8 Ajsagaliev S.A. (1980) Analiz i sintez avtonomnyh nelinejnyh sistem avtomaticheskogo upravlenija [Analysis and synthesis of autonomous nonlinear automatic control systems]. Alma-Ata, Nauka KazSSR. 244. (In Russian)

9 Letov A.M (1962). Ustojchivost' nelinejnyh reguliruemyh system [Stability of nonlinear regulated systems]. M.: Fizmatgiz. 403. (In Russian)

10 Malkin I.G.( 2017) Teorija ustojchivosti dvizhenija [Theory of stability of motion]. URSS.: Izd.4. 432. (In Russian)

11 Fedorjuk M.V. (2015) Asimptoticheskie metody dlja linejnyh obyknovennyh differencial'nyh uravnenij [Asymptotic methods for linear ordinary differential equations].URSS.: Librokom. 390. (In Russian) 